\title{
O enfermeiro frente a atenção à saúde de mulheres vítimas de violência sexual: uma revisão integrativa
}

\author{
The nurse front of the health care of women victims of sexual violence: an integrative review \\ El frente enfermero de la salud de mujeres víctimas de violência sexual: una revisión integrativa
}

Recebido: 26/12/2021 | Revisado: 31/12/2021 | Aceito: 08/01/2022 | Publicado: 11/01/2022

\author{
Samara de Sousa Barbosa \\ ORCID: https://orcid.org/0000-0003-3924-913X \\ Faculdade Ieducare, Brasil \\ E-mail: samara.samayra@hotmail.com \\ David Gomes Araújo Júnior \\ ORCID: https://orcid.org/0000-0003-0755-2118 \\ Faculdade Ieducare, Brasil \\ E-mail: david@fied.edu.br \\ Jordânia Marques de Oliveira Freire \\ ORCID: https://orcid.org/0000-0002-3412-8964 \\ Faculdade Ieducare, Brasil \\ E-mail: jordania@fied.edu.br \\ Iasmin Cunha Maranguape Araújo \\ ORCID: https://orcid.org/0000-0002-2756-846X \\ Faculdade Ieducare, Brasil \\ E-mail: iasminmaranguape@ fied.edu.br
}

\begin{abstract}
Resumo
Caracterizar a atuação da enfermagem na atenção à saúde das mulheres que sofrem violência sexual e discutir sua performance no contexto preventivo a partir das evidências na literatura científica. Revisão integrativa de literatura, com busca dos estudos primários publicados de 2015 a 2021, realizada nas bases de dados eletrônicas LILACS, MEDLINE, BDENF e IBECS nos meses de maio e junho de 2021. As buscas foram obtidas pelo cruzamento, nas bases de dados, dos seguintes descritores e palavras: "violência sexual", "enfermagem", "assistência de enfermagem", correlacionados e de forma isolada. Foram incluídos 11 artigos e, a análise indicou que as evidências disponíveis na literatura reportam identificação de casos da violência sexual e o desempenho da assistência, percepção do enfermeiro quanto à abordagem a mulheres vítimas de violência e aspectos das políticas públicas e a reflexão acerca da sua implementação. A atuação da enfermagem frente atenção a mulheres vítima de violência sexual ainda apresenta desafios e necessidade de adequações, tanto por parte dos profissionais quanto das instituições que ofertam este tipo de serviço. Paralelo a isso ainda também é necessário avançar no acolhimento das vítimas, pois os estudos mostram a magnitude de fatores que dificultam o acesso delas ao serviço de saúde.
\end{abstract}

Palavras-chave: Assistência de enfermagem; Enfermagem; Saúde da mulher; Violência sexual.

\begin{abstract}
To characterize the role of nursing in health care for women who suffer sexual violence and discuss its performance in the preventive context based on evidence in the scientific literature. Integrative literature review, with a search for primary studies published from 2015 to 2021, carried out in the electronic databases LILACS, MEDLINE, BDENF and IBECS in May and June 2021. The searches were obtained by crossing, in the databases, of the following descriptors and words: "sexual violence", "nursing", "nursing care", correlated and isolated. Eleven articles were included, and the analysis indicated that the evidence available in the literature reports the identification of cases of sexual violence and the performance of care, nurses' perception regarding the approach to women victims of violence and aspects of public policies and the reflection on their Implementation. The role of nursing in care for women victims of sexual violence still presents challenges and the need for adjustments, both on the part of professionals and institutions that offer this type of service. Parallel to this, it is also necessary to advance in the reception of victims, as studies show the magnitude of factors that hinder their access to the health service.
\end{abstract}

Keywords: Nursing care; Nursing; Women's health; Sexual violence.

\section{Resumen}

Caracterizar el rol de la enfermería en el cuidado de la salud de las mujeres que sufren violencia sexual y discutir su desempeño en el contexto preventivo con base en la evidencia en la literatura científica. Revisión integrativa de la literatura, con búsqueda de estudios primarios publicados de 2015 a 2021, realizada en las bases de datos electrónicas 
LILACS, MEDLINE, BDENF e IBECS en mayo y junio de 2021. Las búsquedas se obtuvieron cruzando, en las bases de datos, los siguientes descriptores y palabras: "violencia sexual", "enfermería", "cuidados de enfermería", correlacionados y aislados. Se incluyeron once artículos y el análisis indicó que la evidencia disponible en la literatura reporta la identificación de casos de violencia sexual y el desempeño de la atención, la percepción de las enfermeras sobre el abordaje de las mujeres víctimas de violencia y aspectos de las políticas públicas y la reflexión sobre su Implementación. El rol de la enfermería en la atención a las mujeres víctimas de violencia sexual aún presenta desafíos y la necesidad de ajustes, tanto por parte de los profesionales como de las instituciones que ofrecen este tipo de servicios. Paralelamente, también es necesario avanzar en la recepción de víctimas, ya que los estudios muestran la magnitud de factores que dificultan su acceso al servicio de salud.

Palabras clave: Atención de enfermeira; Enfermería; La salud de la mujer; Violencia sexual.

\section{Introdução}

A violência em todas as suas proporções e tipos contra o ser humano pode estar presente na maioria da vida das pessoas independentemente da idade e em variados graus de instrução, cultura, classe social e religião. Entre os tipos de violência existentes encontra-se a violência sexual que também é denominada como violência de gênero, por estar relacionada a fundamentação do poder relacionado ao sexo, sendo as mulheres a maioria que de forma direta vivencia esse ato. Este tipo de violência acontece em praticamente todo país, com os mais diversos tipos de regime político e econômico (Meireles, 2016).

De acordo com o Ministério da Saúde do Brasil (2018), a violência sexual é definida como "todo ato sexual, tentativa de consumar um ato sexual ou insinuações sexuais indesejadas, ou ações para comercializar ou usar de qualquer outro modo a sexualidade de uma pessoa por meio da coerção por outra pessoa, independentemente da relação desta com a vítima, em qualquer âmbito, incluindo o lar e o local de trabalho".

Um estudo com 991 participantes caracterizou a violência sofridas por mulheres fundamentados nos instrumentos da Organização Mundial da Saúde (OMS), mostrando que a prevalência de violência, ao longo da vida, foram: psicológica 57,6\%; física $39,3 \%$ e sexual $18,0 \%$. Ainda, as mulheres com até oito anos de estudo, cujas mães sofreram violência por parceiro íntimo, fizeram uso de drogas e foram acometidas pela violência sexual na infância, a prevalência foi maior nas três violências (Santos et al., 2020). Dentre os tipos de violência contra as mulheres, a sexual, embora não seja o tipo mais frequente, em geral, é vista como aquela que pode causar consequências mais impactantes que as demais.

Em decorrência da alta taxa existente desse tipo de violência fez-se necessário a criação de uma legislação específica para respaldo técnico e de conduta, por meio deste fator, assim foi instituída a criação da lei federal 10.778/2003 que estabelece a notificação compulsória em território nacional, dos casos de violência contra a mulher, sendo ela atendida no serviço de saúde público ou privado (Pinto et al., 2017).

A enfermagem enquanto sua prática social, através da suspeita e/ou confirmação de um caso de violência contra a mulher, por intermédio do respaldo técnico e legal deve estabelecer uma linha de cuidado que inclui a notificação do caso. A notificação é um registro sistemático, constituído em um formulário específico, sua execução é necessária para a alimentação de dados epidemiológicos. Ainda que seja estabelecida a responsabilidade legal para a realização da notificação compulsória, é inegável a prevalência da subnotificação, e isso correlaciona-se ao desconhecimento dos profissionais da saúde acerca de sua competência legal (Acosta et al., 2017).

Um dos primeiros atendimentos que a vítima busca é o serviço de saúde portanto, os princípios bioéticos devem ser assegurados como fonte norteadora para a assistência com enfoque em suas ações e suas práticas. $\mathrm{O}$ acolhimento a mulher/pessoa vítima de violência deve advir durante todo o processo de construção do cuidado, devendo ocorrer de forma reservada e durante esse processo o profissional deve atentar-se a situações que possam coibir o relato da vítima naquele momento (Paraná, 2017).

O serviço de Atenção Básica a Saúde assim como também o profissional da enfermagem dentro desse contexto de atenção à saúde, merece uma atenção singular, ao tratar das suas potencialidades quanto as suas intervenções, pois este serviço 
tem maior ênfase e prioriza uma assistência na qual o enfoque é a promoção à saúde e a prevenção de agravos. Sendo assim capaz de desenvolver ações primordiais para entender e atender esse problema em toda sua complexidade. $O$ desenvolvimento das ações na abordagem do problema tem um dimensionamento complexo fazendo assim a necessidade de uma abordagem multidisciplinar e intersetorial do cuidado (Silva et al., 2017).

As instituições de saúde envolvidas no atendimento à esses tipos de casos devem dispor de capacitação, não obstante cada unidade a depender do nível de atenção de complexidade e dos recursos disponíveis. O atendimento imediato de emergência realizado pela equipe de enfermagem no âmbito preventivo, desenvolve medidas protetivas que visam a redução dos agravos à saúde da mulher como: anticoncepção de emergência, profilaxia de IST/HIV e hepatites, incluindo a interrupção da gravidez (Brasil, 2020).

Confere-se através da problemática exposta hipótese, de que a violência sexual é considerada um problema de saúde pública grave, portanto infere-se como relevância do estudo a necessidade do aprofundamento temático como forma de aprimoramento da atenção à saúde da mulher na implementação do cuidado de enfermagem. Além do que o profissional enfermeiro contribui não apenas para prevenção, mas também na veiculação de informação e/ou discussão acerca do assunto envolvido.

Esta pesquisa, portanto, se justifica pelo crescimento de casos de crime contra mulheres existente no país e no mundo, visto que é uma realidade que deve ser enfrentada e necessita de uma abrangência fidedigna na sociedade através de órgãos públicos principalmente o setor saúde. A partir desse contexto, o presente estudo tem como objetivo caracterizar a atuação da enfermagem na atenção à saúde das mulheres que sofrem violência sexual e discutir sua performance no contexto preventivo a partir das evidências na literatura científica.

\section{Metodologia}

Trata-se de uma revisão integrativa de literatura, norteada pela questão de pesquisa: De que forma o enfermeiro pode atuar para o desenvolvimento de ações que visam a prevenção e o tratamento de agravos durante a assistência a mulheres vítimas de violência sexual? Para a formulação da pergunta de revisão, utilizaram-se os elementos da estratégia PICO (população/paciente - P: mulheres vítimas de violência sexual; intervenção - I: assistência de enfermagem; comparação - C: não se aplica; e "outocomes" (desfecho) - O: evidência).

A produção de dados compreendeu a busca nas bases de dados eletrônicas Literatura Latino Americana e do Caribe em Ciências da Saúde (LILACS), Banco de Dados em Enfermagem - Bibliografia Brasileira (BDENF), Índice Bibliográfico Español en Ciencias de la Salud (IBECS) sendo acessadas via portal Biblioteca Virtual em Saúde (BVS) e Medical Literature and Retrivial System Online (MEDLINE), via PubMed. Realizou-se busca dos descritores junto ao DeCS (Descritores em Ciências da Saúde) e MeSH (Medical Subject Headings). A estratégia de busca na BVS incluiu "violência sexual" AND "enfermagem" OR "assistência de enfermagem" [descritor de assunto]. Para a MEDLINE, utilizou-se "sexual violence" AND "nursing" OR "nursing care".

Como critérios de inclusão, foram consideradas todas as produções primárias que abordassem o tema proposto, respondessem à pergunta de pesquisa e estivessem disponíveis na íntegra online e gratuitamente. A fim de compreender produções nacionais e internacionais os idiomas inglês, espanhol e português forma comtemplados. O levantamento dos estudos ocorreu em maio e junho de 2021. Para a realização da seleção dos estudos, a fim de minimizar eventuais viés de seleção, que consiste em erro de interpretação dos resultados, estabeleceu-se a presença de dois revisores.

Com acesso nas fontes de dados para obtenção das evidências foram identificados 10.717 estudos total ao aplicar o cruzamento definido, para melhor alcance dos estudos que correspondem a temática em específico foram aplicados filtros de seleção como forma de estabelecer prioridades no decorrer da pesquisa. 
Inicialmente, realizou-se a seleção dos estudos completos e disponíveis (5.560), frente ao extenso número de publicações, adotou-se o critério de exequibilidade analítica, que considerou apenas artigos publicados nos últimos cinco anos (2016 a 2021), contabilizando assim 2.708, com a leitura dos títulos e resumos, sendo posteriormente incluídas produções que atenderam aos critérios de inclusão, totalizando 11 artigos selecionados (Figura 1).

Figura 1 - Fluxograma das etapas que compõem o processo de busca e seleção dos estudos.

\begin{tabular}{|c|c|c|c|c|}
\hline \multicolumn{5}{|c|}{$\mathbf{1}^{\mathrm{a}}$ Etapa } \\
\hline \multicolumn{5}{|c|}{ Estratégia de busca: "Violência sexual" AND enfermagem OR "assistência de enfermagem". } \\
\hline \multicolumn{5}{|c|}{$x^{2}$} \\
\hline \multicolumn{5}{|c|}{ Total de artigos/estudos identificados por meio da pesquisa em base de dados: (10.717) } \\
\hline \multicolumn{5}{|c|}{ 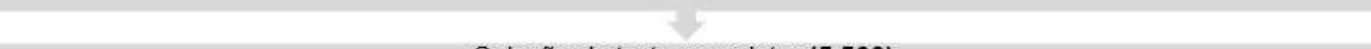 } \\
\hline \multicolumn{5}{|c|}{ Seleção de texto completo: $\mathbf{( 5 . 5 6 0 )}$} \\
\hline \multicolumn{5}{|c|}{ Após aplicacão de filtro de corte temporal últimos 5 anos:(2.708) } \\
\hline \multicolumn{5}{|c|}{ Após aplicação de filtro de corte temporal últimos 5 anos:(2.708) } \\
\hline \multicolumn{5}{|c|}{ Artigos/estudos identificados através da seleção de idiomas. (2.651) } \\
\hline \multicolumn{5}{|c|}{ 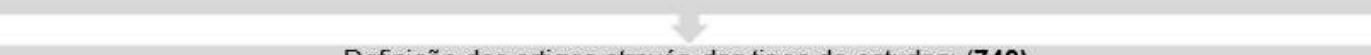 } \\
\hline \multicolumn{5}{|c|}{ Definição dos artigos através dos tipos de estudos: (748) } \\
\hline \multicolumn{5}{|c|}{$2^{\text {a }}$ Etapa } \\
\hline \multicolumn{5}{|c|}{ QUANTO A ELEGIBILIDADE: } \\
\hline $\begin{array}{c}\text { Bases de } \\
\text { dados: (579) }\end{array}$ & $\begin{array}{l}\text { Filtragem dos } \\
\text { assuntos } \\
\text { principais: (592) }\end{array}$ & $\begin{array}{l}\text { Artigos/estudos } \\
\text { identificados } \\
\text { permaneceram } \\
\text { para a leitura de } \\
\text { títulos e } \\
\text { resumos: (579) }\end{array}$ & $\begin{array}{l}\text { Artigos/estudos } \\
\text { identificados } \\
\text { permaneceram } \\
\text { para a leitura } \\
\text { integral: (39) }\end{array}$ & $\begin{array}{l}\text { Artigos/estudos } \\
\text { foram } \\
\text { selecionados: } \\
11 \text { artigos }\end{array}$ \\
\hline
\end{tabular}

Fonte: Autores (2021).

A análise dos resultados perpassou pelo desenvolvimento da síntese dos estudos selecionados e realização de comparações acerca dos principais resultados que respondem à pergunta de revisão, com destaque às diferenças e semelhanças entre os estudos. Assim, a análise e síntese dos resultados desta revisão integrativa, na forma descritiva, foram pautadas nos dados que responderam à pergunta de revisão.

\section{Resultados}

A pesquisa em questão caracteriza-se através da interlocução e análise dos estudos expostos no quadro de distribuição e triagem de artigos selecionados (Quadro 1), onde enaltecem a avaliação e o desempenho do profissional enfermeiro no desenvolvimento da assistência voltado a vítimas de violência sexual. Os estudos correspondem as seguintes características descritas no quadro abaixo. 
Quadro 1 - Caracterização dos estudos selecionados para revisão.

\begin{tabular}{|c|c|c|c|c|c|}
\hline & ANO & REVISTA/ JORNAL DE PUBLICAÇÃO & BASE DE DADOS & TIPO DE ESTUDO & LOCAL / PÁIS \\
\hline A1 & 2018 & PAN AFRICAN MEDICAL JOURNAL & MEDLINE & ESTUDO DE AVALIAÇÃO & ÁFRICA \\
\hline A2 & 2018 & REV. SAÚDE COLETIVA & MEDLINE & ESTUDO DE PREVALENCIA & BRASIL \\
\hline A3 & 2018 & CADERNO DE SAÚDE PÚBLICA & LILACS & ESTUDO DE RASTREAMENTO & BRASIL \\
\hline A4 & 2020 & SAÚDE E SOCIEDADE & LILACS & PESQUISA QUALITATIVA & BRASIL \\
\hline A5 & 2015 & REV. COTIGARE ENFERMAGEM & BEDENF/LILACS & ESTUDO DE PREVALÊNCIA & BRASIL \\
\hline A6 & 2020 & SOUTH AFRICAN MEDICAL JOURNAL & MEDLINE & PESQUISA QUALITATIVA & AFRICA DO SUL \\
\hline A7 & 2018 & REV. DE ENERMAGEM UFPE ONLINE & BEDENF & ESTUDO OBSERVACIONAL & BRASIL \\
\hline A8 & 2020 & REV. PLOS ONE & MEDLINE & PESQUISA QUALITATIVA & EUA \\
\hline A9 & 2020 & REV. NURSING & BEDENF/LILACS & PESQUISA QUALITATIVA & BRASIL \\
\hline A10 & 2015 & REV. BRASILEIRA EPIDEMIOLOGIA & LILACS & ESTUDO DE PREVALENCIA & BRASIL \\
\hline A11 & 2017 & REV. CIÊNCIA E SAÚDE COLETIVA & SCIELO & ESTUDO DE AVALIAÇÃO & BRASIL \\
\hline
\end{tabular}

Fonte: Autores (2021).

A seguir apresenta-se os dados relacionados aos 11 estudos selecionados e inclusos nesta revisão, os artigos expostos no quadro síntese (Quadro 2) apresentam os principais dados dos estudos baseando-se na análise crítica e detalhada de cada um.

Quadro 2 - Síntese das evidências dos estudos selecionados para revisão.

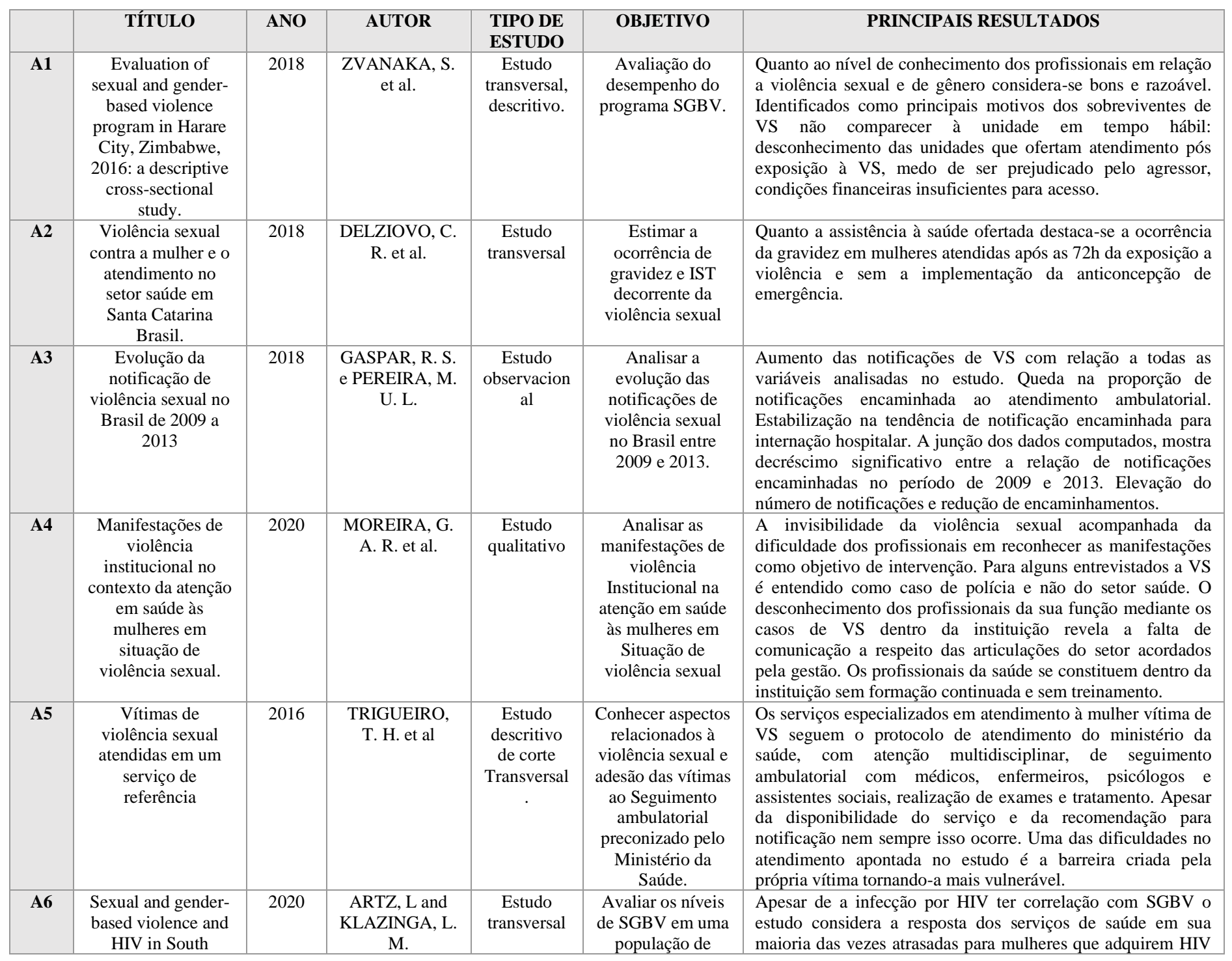




\begin{tabular}{|c|c|c|c|c|c|c|}
\hline & $\begin{array}{c}\text { Africa: An HIV } \\
\text { facility-based study }\end{array}$ & & & & $\begin{array}{c}\text { pacientes de uma } \\
\text { unidade de HIV } \\
\text { no SA. }\end{array}$ & $\begin{array}{l}\text { através da consumação da VS, porém devido à falta de } \\
\text { informação e dados persistentes a prevalência desses } \\
\text { indicadores são mal definidos, existindo razoes especificas } \\
\text { para que isso ocorra: falta de treinamento, visão de que a } \\
\text { triagem para SGBV não faz parte ou é importante para o } \\
\text { processo de ATV ou está além do alcance do papel do } \\
\text { profissional. }\end{array}$ \\
\hline A7 & $\begin{array}{c}\text { Perfil das } \\
\text { notificações sobre } \\
\text { violência sexual }\end{array}$ & 2018 & $\begin{array}{l}\text { BATISTA, V. } \\
\text { C. et al. }\end{array}$ & $\begin{array}{l}\text { Estudo } \\
\text { quantitativo } \\
\qquad, \\
\text { transversal, } \\
\text { de base } \\
\text { secundária }\end{array}$ & $\begin{array}{l}\text { Traçar o perfil dos } \\
\text { casos de violência } \\
\text { sexual notificados } \\
\text { e atendidos em } \\
\text { um hospital de } \\
\text { referência. }\end{array}$ & $\begin{array}{l}\text { A VS prevalece na idade adulta associadas a violência } \\
\text { psicológica a maioria das ocorrências perpetradas nas } \\
\text { residências e em vias públicas com uma porcentagem } \\
\text { considerável em relação ao grau de parentesco sendo sua } \\
\text { maioria desconhecido, amigo/conhecido. Quanto ao } \\
\text { atendimento realizado as vítimas de violência sexual a maioria } \\
\text { recebeu profilaxia para HIV. }\end{array}$ \\
\hline A8 & $\begin{array}{l}\text { Understanding the } \\
\text { healthcare provider } \\
\text { response to sexual } \\
\text { violence in Ghana: } \\
\text { A } \\
\text { situational analysis }\end{array}$ & 2020 & $\begin{array}{l}\text { CANNON, L. } \\
\text { M. et al. }\end{array}$ & $\begin{array}{c}\text { Estudo } \\
\text { descritivo }\end{array}$ & $\begin{array}{l}\text { Análise } \\
\text { situacional para } \\
\text { entender melhor a } \\
\text { resposta do } \\
\text { profissional de } \\
\text { Saúde à violência } \\
\quad \text { sexual. }\end{array}$ & $\begin{array}{l}\text { A resposta ao atendimento à vítima de VS torna-se limita } \\
\text { devido as instalações de acolhimento e procedimento } \\
\text { insuficientes assim como também por apresentarem uma } \\
\text { variação quanto à conduta a ser realizada. Somado ao fator de } \\
\text { que a minoria dos profissionais da instituição recebera } \\
\text { treinamento para este tipo de atendimento. }\end{array}$ \\
\hline A9 & $\begin{array}{l}\text { Percepções de } \\
\text { enfermeiros da } \\
\text { atenção primária no } \\
\text { atendimento à } \\
\text { mulheres vítimas de } \\
\text { violência sexual. }\end{array}$ & 2020 & $\begin{array}{l}\text { MOTA, J. A. e } \\
\text { AGUIAR, S. R. }\end{array}$ & $\begin{array}{l}\text { Estudo } \\
\text { descritivo } \\
\text { exploratóri } \\
\text { o, com } \\
\text { abordagem } \\
\text { qualitativa. }\end{array}$ & $\begin{array}{l}\text { Analisar a } \\
\text { percepção dos } \\
\text { enfermeiros sobre } \\
\text { o atendimento às } \\
\text { mulheres vítimas } \\
\text { de violência } \\
\text { sexual na atenção } \\
\text { primaria. }\end{array}$ & $\begin{array}{l}\text { Diante da realidade existente e da diferença de gênero, os } \\
\text { profissionais ao realizar o atendimento a essas vítimas } \\
\text { declaram sentimento de empatia, pena, frustação, e alguns de } \\
\text { alguma forma a dúvida acompanhada de certos julgamentos. A } \\
\text { empatia permite um cuidado sensível e acolhedor, já a sua } \\
\text { ausência ocasiona mal atendimento e afastamento da mulher } \\
\text { ao serviço de saúde. No que se refere a atuação do enfermeiro } \\
\text { nota-se o despreparo o que pode resultar em encaminhamento } \\
\text { e subnotificação dos casos. }\end{array}$ \\
\hline A10 & $\begin{array}{l}\text { Preenchimento da } \\
\text { notificação } \\
\text { compulsória em } \\
\text { serviços de saúde } \\
\text { que atendem } \\
\text { mulheres que } \\
\text { sofrem violência } \\
\text { sexual }\end{array}$ & 2016 & $\begin{array}{l}\text { SOUSA, M. H. } \\
\quad \text { et al. }\end{array}$ & $\begin{array}{l}\text { Estudo com } \\
\text { abordagem } \\
\text { mista }\end{array}$ & $\begin{array}{l}\text { Avaliar a } \\
\text { proporção de } \\
\text { serviços de saúde } \\
\text { que preenchem a } \\
\text { notificação } \\
\text { compulsória e } \\
\text { quais os principais } \\
\text { obstáculos para o } \\
\text { preenchimento de } \\
\text { tal documento }\end{array}$ & $\begin{array}{l}\text { Mais da metade dos participantes responsáveis pelo } \\
\text { atendimento dentro dos serviços de saúde que prestavam } \\
\text { atendimento de emergência às mulheres que sofreram } \\
\text { violência sexual eram enfermeiros, a maioria dos serviços } \\
\text { afirmaram preencher a ficha de notificação. Porém foram } \\
\text { relatados pelos participantes motivos para o não } \\
\text { preenchimento da ficha de notificação como: ficha muito } \\
\text { extensa, dificuldade para obtenção de informações e referente } \\
\text { ao despreparo dos profissionais. Assim como também foi } \\
\text { relatado o desconhecimento para qual órgão enviar a ficha } \\
\text { preenchida. }\end{array}$ \\
\hline A11 & $\begin{array}{l}\text { Políticas públicas } \\
\text { de proteção à } \\
\text { mulher: avaliação } \\
\text { do atendimento em } \\
\text { saúde de vítimas de } \\
\text { violência sexual }\end{array}$ & 2017 & $\begin{array}{l}\text { PINTO, L. S.S. } \\
\text { et al. }\end{array}$ & $\begin{array}{c}\text { Estudo } \\
\text { exploratóri } \\
\text { o e } \\
\text { descritivo. }\end{array}$ & $\begin{array}{l}\text { Avaliar as } \\
\text { políticas públicas, } \\
\text { a legislação de } \\
\text { proteção à mulher } \\
\text { e os atendimentos } \\
\text { de saúde às } \\
\text { vítimas de } \\
\text { violência sexual. }\end{array}$ & $\begin{array}{l}\text { Através da análise do estudo e das políticas públicas } \\
\text { implementadas tem-se como resultado a evolução histórica do } \\
\text { contexto do atendimento às vítimas de violência sexual, que } \\
\text { através das leis implementadas, diretrizes de atendimento e } \\
\text { condutas elegida e construídas através do conjunto do poder } \\
\text { público e movimentos feministas existe atualmente maior } \\
\text { aparato para acolhimento dessas mulheres, mesmo com a } \\
\text { existências de algumas leis ineficientes. }\end{array}$ \\
\hline
\end{tabular}

Fonte: Autores (2021).

\section{Discussão}

Em relação a análise dos resultados apresentados através da exposição dos dados na presente revisão integrativa, como forma de elucidar informações significativas que corroboram para o estudo da temática da pesquisa a seguir será apresentada a discussão dos estudos analisados de acordo com a literatura em três categorias.

\section{Identificação de casos da violência sexual e o desempenho da assistência.}

De antemão pode-se fazer uma ênfase na questão "violência sexual" como uma conjunção significativa quando se transmite para o cuidado e a assistência de enfermagem. Ao avaliar esta assistência dentro de um programa voltado para a assistência à violência sexual baseada no gênero, onde a maioria dos profissionais ali existentes eram enfermeiros infere de forma sucinta que, no decorrer da avaliação foi identificado falhas com relação direta ao fator da falta de aperfeiçoamento e consequentemente a falta de conhecimento (Sithole, 2018). 
A assistência de enfermagem às mulheres que sofrem violência principia pelo acolhimento, no entanto finalizada quando ela é encaminhada, contrariando a ideia dos serviços de saúde que trabalham em rede (Silva e Ribeiro, 2020). A assistência de enfermagem pode ser requerida e protocolada por meio da solicitação nos serviços responsáveis pelo atendimento, é elencada por meio de condutas direcionadas à quesitos biológicos. O manejo do cuidado das lesões de pele das vítimas violentadas é tarefa e responsabilidade da enfermagem, na qual contribui para melhora física. A realização de curativos é uma atribuição incluída neste cuidado quando é ferimento exposto/aberto resultante da violência (Santos et al., 2021).

Acerca do assunto em questão é notório que a assistência de enfermagem possui um embasamento teórico satisfatório, porém a sua implementação passa por diversas fragmentações tornando-a enfraquecida quando existe uma deficiência considerável na execução de medidas burocráticas, com influência da precariedade na educação continuada para colaboradores da área assistencial. Ou seja, a identificação de casos de VS sofre negligência, assim como também a implementação da assistência considerando tais fatores.

O fator violência sexual já é um agravante importante para a saúde pública, trazendo isso para a conjunção da violência sexual e gênero enfatiza-se de forma considerável que a assistência em si deveria de ser graduada como satisfatória. A enfermagem tem predominância em humanização e nesta oferta de cuidado não pode ser diferente, no entanto a realidade desses profissionais em sua grande maioria é deficiente e insalubre dificultando a execução dele.

Delziovo et al., (2018), considera uma percepção voltada para a participação do enfermeiro na implementação do cuidado como sendo a categoria que mais participa ativamente do acolhimento às sobreviventes da VS. Este estudo assemelhase com o citado anteriormente quanto as problemáticas apresentadas. Contempla a importância de uma assistência adequada traz dados importantes como: ocorrência de gravidez como consequência da violência sexual foi de $7,6 \%$. Nesta amostra, a maioria possuía idade entre 10 e 14 anos (10,8\%) e baixa escolaridade (13,3\%).

A presença frequente do profissional enfermeiro (a) no processo do cuidar frente às vítimas nos serviços de saúde é um dos fatores que coopera para a extensão deste elo da relação e diálogo profissional/usuário, viabilizando o alcance de informações que outros da equipe multiprofissional não têm acesso por não ficarem tão próximos e por tanto tempo. Dessa forma, o enfermeiro(a) acaba sendo, na maioria das vezes, realiza o primeiro contato profissional de apoio à vítima. O registro na ficha compulsória, sobre a violência, é atividade de competência do Enfermeiro (Silva et al., 2021).

Considerando a hipótese levantada para o fundamentação teórica e analítica deste estudo que tem a VS como problema de saúde pública, é notório que os fatores sociais implicam diretamente na conduta não apenas do profissional enfermeiro assim como também no sobrevivente de VS. Dado isso, também sabemos que o enfermeiro é um profissional primordial para o desenvolvimento de uma assistência com enfoque na prevenção e detecção de problemas que interferem na implementação do cuidado, porém ainda é recorrente a semelhança da problemática encontrada no desenvolvimento da assistência para este evento em específico. Tendo como maior agravante uma resposta não positiva a respeito da do conhecimento teórico e prático na implementação da assistência no contexto da VS.

\section{Análise acerca da percepção do enfermeiro quanto à abordagem a mulheres vítimas de violência.}

A pesquisa procurou responder de que forma o enfermeiro pode atuar para o desenvolvimento de ações que visam a prevenção e o tratamento de agravos durante a assistência a mulheres vítimas de VS. É importante evidenciar os principais fatores que de fato interferem na assistência em saúde como um todo, assim como também de imediato entender que o acesso à saúde tem grande importância no desfecho e evolução do cuidado a essas pessoas vítimas de VS.

De acordo com Moreira et al (2020), inúmeros fatores relatados por seus entrevistados corroboram para uma análise situacional e para construção de uma ideia a respeito do assunto. Onde expõe o fator da invisibilidade da VS por parte dos 
profissionais dada pela dificuldade em reconhecer as manifestações como objeto de intervenção, onde ocorre a fragilidade da relação entre o profissional e a vítima.

As instituições qualificadas como referência contribuem para maior Empoderamento assistencial do profissional ali vinculado a mesma, em contrapartida, é importante destaca que a assistência à sobreviventes da violência sexual, não depende apenas do profissional que presta a assistência mais também da participação ativa e esclarecida da vítima. Onde sabemos que nem sempre isso ocorrerá devido a instalação de algumas barreiras por parte dela, na qual reflete diretamente na resposta à abordagem, tratamento e recuperação.

No que se refere ao desempenho da assistência analisado anteriormente e a percepção do profissional, se tem basicamente a resposta de que esse atendimento muitas vezes encontra-se prejudicada e limitada também por fatores físicos estruturais, como instalações de acolhimento e a disposição de recursos e insumos. Tornando os procedimentos insuficientes, que repercute em uma variação quanto a conduta ofertada (Cannon, 2020).

A abordagem a esta temática ela transcende aos fatores específicos da assistência, o enfermeiro em sua maioria das vezes entende sua importância e sua relevância, não obstante é necessário desvencilhar-se de fatores intrínsecos/pessoais que podem vir a interferir no acolhimento a essas sobreviventes vítimas de VS.

A percepção do enfermeiro a respeito do atendimento em si, explana a existência de uma diversidade de opiniões no que se refere ao desenrolar da assistência e ao enfrentamento do evento. Onde muitos apresentam a ideia de que para um bom atendimento é fundamental a empatia, mas também muitas vezes existe a frustração quando não é possível o atendimento adequado independente do fator ao qual ocasiona isto.

\section{Análise dos aspectos das políticas públicas e a reflexão acerca da sua implementação.}

As políticas públicas voltadas para a saúde da mulher são consideradas um avanço histórico e de extrema importância para o desenvolvimento do seu papel na sociedade, visto que a diferenciação de gênero faz necessário o conhecimento e a implementação das mesmas. No entanto a evolução da assistência à saúde da mulher de forma integral desenvolve maior autonomia, melhor aplicabilidade assim como também desenvolve maior conhecimento por parte delas quanto ao fator saúdedoença e maior reconhecimento dos seus direitos.

Pinto et al. (2017), a legislação de proteção à mulher tem como resultado a evolução histórica do atendimento a vítimas de violência sexual, dada através da implementação leis e das diretrizes de atendimentos e condutas, construídas pelo acolhimento a essas mulheres, também através do interesse do poder público com forte influência dos movimentos feministas existentes. Mesmo com toda esta influência das políticas de atenção à saúde da mulher ainda existe leis consideradas ineficientes, não apenas no objetivo de punição mais também dentro de todo o processo que transcorre o eixo burocrático que complementa a avaliação da assistência e a contemplação de dados epidemiológicos.

Ao analisar o desenvolvimento do atendimento à sobreviventes da violência sexual e o seguimento do protocolo de atendimento do Ministérios da Saúde, com foco em uma prestação de serviço de atendimento multidisciplinar é observado que apesar dessa disponibilidade de serviço e da recomendação para a notificação, nem sempre é realizada (Trigueiro, 2015).

Gaspar e Pereira (2018), aponta que no ano de 2009 e 2013 ocorreu um aumento de notificação de violência sexual com foco no estupro e uma redução na proporção de encaminhamento. Isto traz uma ideia antagônica a análise apresentada pelos demais autores, podendo estar relacionada a diversos fatores como: educação permanente, maior conhecimento por parte dos profissionais e a preservação da longitudinalidade do cuidado.

A notificação é um elemento importante no que diz respeito aos indicadores de saúde, considerados subsídios que visam a avaliação situacional de dados epidemiológicos, com o intuito de elaborar e implementar ações para melhor desempenho da assistência. Ao avaliar a proporção dos serviços de saúde que realizam o preenchimento da ficha de notificação 
e suas dificuldades foi identificado que a maioria dos serviços a realização. A maior parte dos profissionais responsáveis pelo atendimento de emergência prestado a vítimas de violência sexual, são os enfermeiros. Estes apontam motivos para o não preenchimento da ficha de notificação, são eles: fichas muito longas, dificuldade para a obtenção de informações e despreparo profissional (Bezerra, 2018).

Ao avaliar os presentes dados foi possível afirmar que a notificação compulsória, o acolhimento e a realização da escuta ativa e de forma adequada são fatores essenciais para a realização da assistência, porém quanto a notificação compulsória faz-se necessário o entendimento da real importância em realizá-la.

Os serviços de saúde perpassam por um período oportuno à reflexão. Os questionamentos surgem do incômodo instigado naqueles que sentem dificuldade sobre discussões no que diz respeito a humanização em saúde com um olhar atento: a população brasileira transpassa por situações de violência quando busca por serviços de saúde. A violência presente internamente nos serviços tornou-se algo tão recorrente que a humanização da saúde surge como reivindicação social para respeitar os direitos da população e como política pública que visa atuar nessa violência (Azeredo \& Schraiber, 2021).

\section{Considerações Finais}

A violência sexual contra a mulher é considerada um problema de saúde pulica grave e a assistência de enfermagem considerada uma intervenção indispensável, para um atendimento humanístico e individualizado, desde que ocorra de forma não empírica e em tempo hábil. Tendo a caracterização destes elementos como foco principal da pesquisa foi possível perceber um misto de informações de acordo com os instrumentos disponíveis para a assistência. Assim como também o envolvimento do nível de instrução do profissional como colaborador para este tipo de assistência.

Dessa forma a atuação da enfermagem frente atenção a mulheres vítima de violência sexual ainda apresenta desafios e necessidade de adequações, tanto por parte dos profissionais quanto das instituições que ofertam este tipo de serviço. Paralelo a isso ainda também é necessário avançar no acolhimento das vítimas, pois os estudos mostram a magnitude de fatores que dificultam o acesso delas ao serviço de saúde. No âmbito da pesquisa ainda há lacunas que precisam ser exploradas, e preenchidas em estudos futuros para a contribuição e explanação do conhecimento. No que se refere as ações de saúde pública é notório que há necessidade de maior abrangência e ampliação da saúde da mulher, no contexto biopsicossocial, assim como também e faz necessário a consolidação da equipe multidisciplinar atuante no fluxo desse tipo de atendimento.

Destarte esta pesquisa contribuiu de forma significativa para melhor conhecimento das teorias aplicadas na assistência em relação a temática, assim como também corrobora para explanação da importância da implementação do cuidado pelo profissional enfermeiro dentro no contexto de promoção e prevenção à saúde das mulheres vulneráveis a violência sexual.

\section{Referências}

Acosta, D. F., Gomes, V. L. d. O., Oliveira, D. C. d., Gomes, G. C., \& Fonseca, A. D. d. (2017). Aspectos éticos e legais no cuidado de enfermagem às vítimas de violência doméstica. Texto contexto - enferm, 3(22). https://doi.org/10.1590/0104-07072017006770015

Arrais Mota, J.., \& Saraiva Aguiar, R. (2020). Percepções de enfermeiros da atenção primária no atendimento í s mulheres vítimas de violência sexual. Nursing (São Paulo), 23(262), 3648-3651. https://doi.org/10.36489/nursing.2020v23i262p3648-3651

Azeredo, Y. N., \& Schraiber, L. B. (2021). Autoridade, poder e violência: um estudo sobre humanização em saúde. Interface (Botucatu), (25). https://doi.org/10.1590/Interface. 190838

Bezerra, J. da F., de Lara, S. R. G., do Nascimento, J. L., \& Barbieri, M. (2018). Assistência à mulher frente à violência sexual e políticas públicas de saúde: revisão integrativa. Revista Brasileira Em Promoção Da Saúde, 31(1). https://doi.org/10.5020/18061230.2018.6544

Brasil (2018). Atenção humanizada às pessoas em situação de violência sexual com registro de informações e coleta de vestígios $1^{a}$ edição - $1^{a}$ impressão. Ministério da Saúde. Secretaria de Atenção à Saúde. Departamento de Ações Programáticas Estratégicas. Coordenação-Geral de Saúde das Mulheres, Brasil. 
Research, Society and Development, v. 11, n. 1, e45611125137, 2022

(CC BY 4.0) | ISSN 2525-3409 | DOI: http://dx.doi.org/10.33448/rsd-v11i1.25137

Brasil (2021). Atenção integral às pessoas com infecções sexualmente transmissíveis (IST). Secretaria de vigilância em saúde. Departamento de doença de condições crônicas e IST, Ministério da Saúde.

Cannon, L. M., Sheridan-Fulton, E. C., Dankyi, R., Seidu, A., Compton, S. D., Odoi, A., Darteh, E. K.M., \& Munro-Kramer, M. L. (2020). Understanding the healthcare provider response to sexual violence in Ghana: A situational analysis. PLoS one, 15(4). https://doi.org/10.1371/journal.pone.0231644

Delziovo, C. R., Coelho, E. B. S., d'Orsi, E., \& Lindner, S. R. (2018). Violência sexual contra a mulher e o atendimento no setor saúde em Santa Catarina Brasil. Ciênc. saúde coletivo, 23 (5). https://doi.org/10.1590/1413-81232018235.20112016

Gaspar, R. S., \& Pereira, M. U. L. (2018). Evolução da notificação de violência sexual no Brasil de 2009 a 2013. Cad. Saúde Pública, 34 (11). https://doi.org/10.1590/0102-311X00172617

Meireles, L. S. P. d. (2016). Uma vida sem violência, um direito de toda mulher: análise da violência contra mulher no Brasil [Doctoral dissertation, Universidade Federal do Rio Grande Do Norte]. Biblioteca Setorial do CCSA. https://repositorio.ufrn.br/bitstream/123456789/36305/2/LiviaSPM _Monografia.pdf

Moreira, G. A. R., Vieira, L. J. E. d. S., Cavalcanti, L. F., Silva, R. M. d., \& Feitoza, A. R. (2020). Manifestações de violência institucional no contexto da atenção em saúde às mulheres em situação de violência sexual. Saude soc., 29(1). https://doi.org/10.1590/S0104-12902020180895

Paraná - CWB (2017). Protocolo para o atendimento às pessoas em situação de violência sexual / SAS. Secretaria de Estado da Saúde do Paraná. Superintendência de Atenção à Saúde. Paraná, Curitiba, Brasil.

Pinto, L. S. S., Oliveira, I. M. P. d., Pinto, E. S. S., Leite, C. B. C., Melo, A. d. N., \& Deus, M. C. B. R. d. (2017). Políticas públicas de proteção à mulher: avaliação do atendimento em saúde de vítimas de violência sexual. Ciênc. saúde colet, 5(22). https://doi.org/10.1590/1413-81232017225.33272016

Santos, I. B. D., Leite, F. M. C., Amorim, M. H. C., Maciel, P. M. A., \& Gigante, D. P. (2020). Violência contra a mulher na vida: estudo entre usuárias da Atenção Primária. Ciência \& Saúde Coletiva, 25(5), 1935-1946.

Silva, N. N. F., Leal, S. M. C., Trentin, D., Vargas, M. A. d. O., Vargas, C. P., \& Vieira, L. B. (2017). Atuação dos enfermeiros da atenção básica a mulheres em situação de violência. Enferm. Foco, 8(3), 70-74. https://doi.org/10.21675/2357-707X.2017.v8.n3.1290.

Sithole, Z., Gombe, N. T., Juru, T., Chonzi, P., Shambira, G., Nsubuga, P., \& Tshimanga, M. (2018). Avaliação do programa de violência sexual e baseada em gênero na cidade de Harare, Zimbábue, 2016: um estudo transversal descritivo. Pan African Medical Journal,31(200). https://doi.org/10.11604/pamj.2018.31.200.14791

Silva, V. G. d., \& Ribeiro, P. M. (2020). Violência contra as mulheres na prática de enfermeiras da atenção primária à saúde. Esc. Anna. Nery, 24(4). https://doi.org/10.1590/2177-9465-EAN-2019-0371

Santos, D., Santos, E., Backes, M., Giacomozzi, A., Gomes, I., \& Kalivala, K. (2021). Assistência de enfermagem às mulheres em situação de violência sexual: revisão integrativa. Revista Enfermagem UERJ, 29(1), e51107. https://doi.org/10.12957/reuerj.2021.51107

Silva, B. K. d. S., Queiroz, P. d. SS, Barbosa, MSN, Sousa, HR d., \& Viana, JA (2021). Assistência de enfermagem às vítimas de violência doméstica no contexto da Covid-19. Revista Científica Multidisciplinar Núcleo do Conhecimento, 6 (11), 106-115.

Trigueiro, T., Merighi, M., Medeiros, A., Ribeiro, C., Mata, N., \& Jesus, M. (2015). Vítimas de violência sexual atendidas em um serviço de referência. Cogitare Enfermagem, 20(2). http://dx.doi.org/10.5380/ce.v20i2.40355 